\title{
Wavelength dependence of light diffusion in strongly scattering macroporous gallium phosphide
}

\author{
W. H. Peeters, ${ }^{1}$ I. M. Vellekoop, ${ }^{1}$ A. P. Mosk, ${ }^{1}$ and A. Lagendijk ${ }^{2}$ \\ ${ }^{1}$ Complex Photonic Systems, Faculty of Science and Technology and MESA + Institute for Nanotechnology, University of Twente, \\ P.O. Box 217, 7500 AE Enschede, Netherlands \\ ${ }^{2}$ FOM-Institute for Atomic and Molecular Physics (AMOLF), Kruislaan 407, 1098 SJ Amsterdam, Netherlands
}

(Received 31 July 2007; published 18 March 2008)

\begin{abstract}
We present time-resolved measurements of light transport through strongly scattering macroporous gallium phosphide at various vacuum wavelengths between $705 \mathrm{~nm}$ and $855 \mathrm{~nm}$. Within this range the transport mean free path is strongly wavelength dependent, whereas the observed energy velocity is shown to be independent of the wavelength. We conclude that microscopic resonances, which can strongly slow down the diffusion process in, e.g., granular $\mathrm{TiO}_{2}$, are absent in macroporous gallium phosphide in the wavelength region of concern.
\end{abstract}

DOI: 10.1103/PhysRevA.77.035803

PACS number(s): 42.25.Dd, 05.60.-k, 61.43.Gt

The propagation of electromagnetic waves in strongly scattering disordered media has received increasing attention $[1,2]$. The research is mainly driven by a need for a deep understanding of the influence of interference on the transport properties of electromagnetic waves. If the mean free path in the medium is much larger than the wavelength, the average intensity in the medium is well described by a diffusion equation. In this diffusive regime, the influence of interference on the transport properties is weak. On the other hand, if the mean free path becomes comparable to the wavelength of the light, the effect of interference will grow stronger and the mean free path will be renormalized to a lower value. Interference increases the probability of a wave to return to the place from which it has been scattered. Consequences of this type of interference are enhanced backscattering [3,4] and Anderson localization of light [5-7].

It is expected that Anderson localization of electromagnetic waves occurs according to the Ioffe-Regel criterion for localization $[6,7]$. This criterion states that Anderson localization sets in if $k_{e} l_{B}<1$, where $k_{e}$ is the effective wave number in the scattering medium and $l_{B}$ is the unrenormalized mean free path. At the transition we get $k_{e} l=0$, where $l$ is the (renormalized) transport mean free path. For $k_{e} l_{B}>1$ the average intensity obeys the diffusion equation with $D$ $=v_{E} l / 3$, where $v_{E}$ is called the energy velocity [8]. Experimental studies on Anderson localization of light have been reported in the microwave regime [9-11], the near infrared regime [12], and the visible regime $[2,13,14]$. Strongly scattering materials in the near infrared and visible regime comprise granular $\mathrm{ZnO}$ [15], granular $\mathrm{BaSO}_{4}$ [15], granular $\mathrm{TiO}_{2}$ [2,15-17], granular GaAs [12], and macroporous gallium phosphide [13,18-22]. Macroporous $\mathrm{GaP}$ is among the strongest scattering materials in the visible regime with $k_{e} l$ $=2.6 \pm 0.2$ at $\lambda_{0}=632 \mathrm{~nm}[18]$.

In this paper we report time-resolved measurements on the transport of light in strongly scattering macroporous GaP. The main goal of our experiments is to study the relationship between the energy velocity and the wavelength. By varying the wavelength of the light we will also vary the transport mean free path over almost a factor of 2, covering a large range close to the mobility edge. Until now, it has been unclear how sensitive the magnitude of the energy velocity is to the wavelength. Earlier, it was demonstrated that micro- scopic resonances can strongly reduce the energy velocity in, e.g., granular $\mathrm{TiO}_{2}[8,17]$. We show that microscopic resonances are absent in macroporous $\mathrm{GaP}$ within a wavelength region between $705 \mathrm{~nm}$ and $855 \mathrm{~nm}$.

Because the energy velocity $v_{E}$ cannot be measured directly, we have measured the diffusion constant $D$ and have used the relation $v_{E}=3 D / l$ to obtain $v_{E}$. We have extracted $D\left(\lambda_{0}\right)$ from time-resolved transmission measurements at several wavelengths between $705 \mathrm{~nm}$ and $855 \mathrm{~nm}$. The transport mean free path $l\left(\lambda_{0}\right)$ has already been determined by Johnson et al. [22] from measurements of the total transmission versus sample thickness. Johnson et al. [22] have also measured the diffusion constant, but solely at a single wavelength $\lambda_{0}$ $=739 \mathrm{~nm}$. We significantly supplement the work of Johnson et al. by measuring the wavelength dependence of the diffusion constant.

Our time-resolved transmission measurements are performed with a pulse interferometer setup [16,22-24]. With this technique we obtain the response to an incoming pulse of the electric field in a transmitted speckle spot in the far field. In our experiments, the incoming pulse is formed by focusing a linearly polarized Gaussian beam onto a $30 \mu \mathrm{m}$ wide spot on the sample. The detected speckle is the linearly polarized component of the transmitted wave in the forward direction (having angular width of a single speckle in the far field). The orientation of the sample is visualized in Fig. 1.

From the time-dependent response of the field in a single speckle spot, we calculate the transmitted intensity $I(t)$, generated by a short impinging pulse $(\sim 150 \mathrm{fs})$. The timedependent transmitted intensity in a single speckle spot will contain mesoscopic fluctuations $[25,26]$. To make the comparison with diffusion theory possible we average the timedependent transmitted intensity over different realizations of the random medium. We performed this ensemble averaging by moving the sample in transverse direction between different measurements by more than the size of the illumination spot. In this way, we have measured the intensity responses of about 40 speckles at each of eight different settings of the wavelength. The ensemble of 40 realizations is large enough to obtain a fairly smooth curve of the time-dependent average transmitted intensity $\langle I(t)\rangle$ (see Fig. 3).

By comparing $\langle I(t)\rangle$ with diffusion theory we extract the 


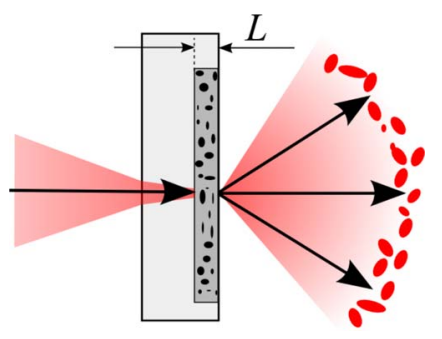

FIG. 1. (Color online) Schematic representation of the profile of a macroporous gallium phosphide $(\mathrm{GaP})$ sample and its orientation in the experimental setup. The homogeneously shaded area of the sample represents the GaP wafer, consisting of bulk GaP. The dotted area of the sample represents the macroporous $\mathrm{GaP}$ layer with thickness $L$. The thick arrows indicate the direction of the light. A far field speckle pattern is formed on the right-hand side of the sample.

diffusion constant of the light in the medium. The pulse response function of the transmitted intensity through a diffusing material in a slab geometry is given by [27]

$$
\begin{aligned}
T(t)= & \frac{-2 \pi D e^{-t / \tau_{a}}}{L_{e}^{2}} \sum_{n=1}^{\infty} n \sin \left(\pi n \frac{l\left(1+z_{1}\right)}{L_{e}}\right) \\
& \times \cos \left(\pi n \frac{L-l\left(1+z_{1}\right)}{L_{e}}\right) e^{-\pi^{2} n^{2} D t / L_{e}^{2}},
\end{aligned}
$$

where $\tau_{a}$ is the absorption time, $D$ is the diffusion constant, $L$ is the sample thickness, $L_{e} \equiv L+l\left(z_{1}+z_{2}\right)$, and $z_{1}, z_{2}$ are the extrapolation ratios at the input side and output side, respectively. It has been shown in previous measurements that the absorption time is much larger than the average transmission time $\tau_{0}$ in these porous GaP samples $[13,20,21]$. Therefore, it is allowed to neglect the influence of absorption when dealing with times in the order of $\tau_{0} \approx L^{2} / 6 D$. Johnson et al. [22] have measured $z_{1}=0.95 \pm 0.1$ and $z_{2}=2.1 \pm 0.3$ from escape function measurements following a technique proposed by Vera and Durian [28], and have obtained $l\left(\lambda_{0}\right)$ from measurements of the total amount of transmitted light as a function of sample thickness (see Fig. 2). We have measured the thickness $L$ with a scanning electron microscope to an accuracy of about $1 \%$.

In order to extract the diffusion constant from the measured time-dependent transmission $\langle I(t)\rangle$, we fit the convolution of Eq. (1) with the impinging pulse to the measured curve. We only use the diffusion constant $D$ and an amplitude normalization factor as fit parameters and we substitute the known values for $L, l, z_{1}$, and $z_{2}$. An example of the measured curve and the theoretical curve is visualized in Fig. 3 . Using just two fit parameters, we obtain excellent agreement between the measured curve $\langle I(t)\rangle$ and the fitted theoretical curve. The fit in this example yields a diffusion constant of $D=32.9 \pm 2.4 \mathrm{~m}^{2} \mathrm{~s}^{-1}$.

The part of the response after $t=8.7 \mathrm{ps}$ is excluded from the fit because this part is affected by a sequence of after pulses caused by internal reflections in the sample substrate. The impinging pulse rings down inside the substrate (the nonporous bulk $\mathrm{GaP}$ wafer) via a diffuse reflection on the rear surface of the porous layer and a specular reflection on

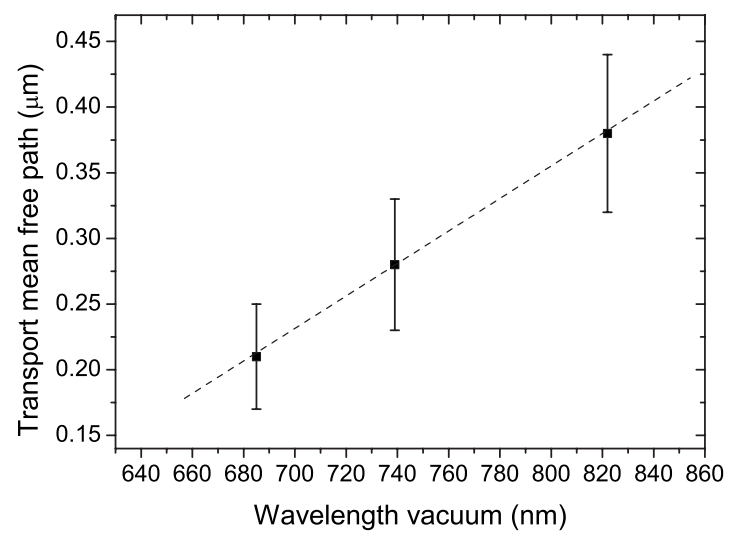

FIG. 2. Transport mean free path of PA-GaP measured at three different vacuum wavelengths by Johnson et al. [22]. The error bars are mutually fully correlated $[22,29]$. The dotted line is a linear regression.

the opposite flat surface of the wafer (see Fig. 1). We expect pulse intervals of about $\Delta t=2 d n_{\mathrm{gr}} / c=8.4 \mathrm{ps}$, where $d$ $=340 \mu \mathrm{m}$ is the substrate thickness and $n_{\mathrm{gr}}=3.7$ is the group velocity in bulk $\mathrm{GaP}$ at $750 \mathrm{~nm}[30,31]$. The after pulses are clearly separated from each other in the time-dependent transmission of a relatively thin porous layer as shown in the inset of Fig. 3. The temporal spacing between the pulses is in agreement with our expectations. The second (and later) after pulses are stretched in the time domain because they contain light that is reflected in lateral directions by two (or more) diffuse reflections on the porous layer. The first after pulse is negligibly stretched in time as only light reflected close to

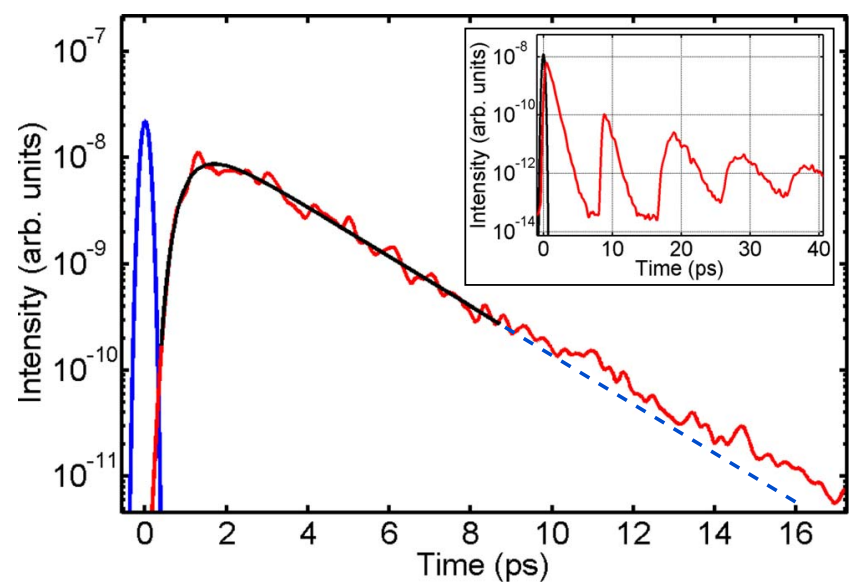

FIG. 3. (Color online) Our measurement of the diffusively transmitted intensity through a $23.6 \pm 0.2-\mu$ m-thick macroporous $\mathrm{GaP}$ sample. The curve is obtained by averaging the intensity response of 44 different speckles at a wavelength of $\lambda_{0}=775 \mathrm{~nm}$. The narrow pulse on the left-hand side is the incident pulse. The smooth line is Eq. (1), derived from diffusion theory with $D=32.9 \mathrm{~m}^{2} \mathrm{~s}^{-1}$. The part of the response after $t=9 \mathrm{ps}$ is affected by an after pulse. The inset shows the average transmitted intensity of a $9.55 \pm 0.16-\mu \mathrm{m}$-thick porous GaP layer. A series of after pulses caused by internal reflections inside the sample substrate is clearly visible. The low-level plateaus at about $10^{-13.5}$ (arbitrary units) are at the noise level. 


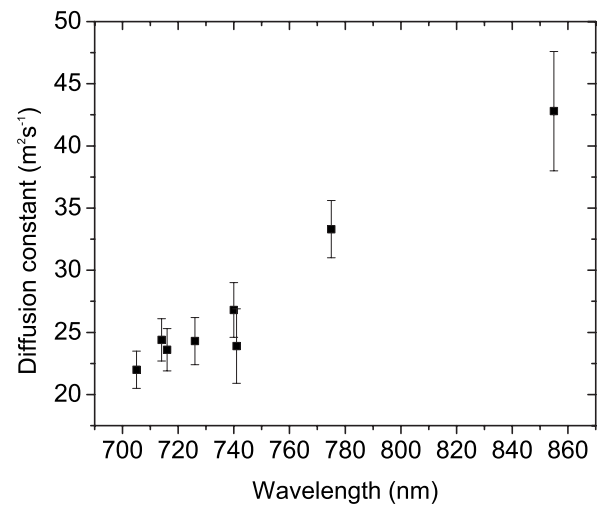

FIG. 4. Our measurement of the wavelength-dependent diffusion constant, extracted from the time-dependent diffuse transmission, of a $48.5 \pm 0.5-\mu \mathrm{m}$-thick sample versus wavelength in vacuum.

the surface normal can pass the detection aperture after one diffuse reflection.

The presence of the after pulses makes it impossible to interpret the long-time part of the transmitted intensity (see Fig. 3). Especially signs of Anderson localization of light, that could manifest themselves at long times [14,32,33] will become difficult to detect. In the present setup, it is impossible to get rid of the after pulses experimentally without changing the samples. Although the pulse interferometer technique is very well suited to detect weak signals at long times, the presence of the after pulses is the limiting factor when it comes to measuring long-time tails.

We have measured the diffusion constant of the macroporous GaP layer at several different wavelengths between $705 \mathrm{~nm}$ and $855 \mathrm{~nm}$. The results are shown in Fig. 4. We observe that a change of only $20 \%$ in wavelength almost doubles the diffusion constant. Johnson et al. [22] have determined the transport mean free path at three different wavelengths, via measurements of the total transmission versus sample thickness (see Fig. 2). Additional fine-meshed measurements of the total transmission versus wavelength, also performed by Johnson et al., indicate a linear relationship between $l$ and $\lambda_{0}$ [22,34]. We have therefore made a linear regression on the three points to obtain $l\left(\lambda_{0}\right)$ between $680 \mathrm{~nm}$ and $860 \mathrm{~nm}$ (see Fig. 2). We use $l\left(\lambda_{0}\right)$ to plot the diffusion constant versus the resulting transport mean free path as shown in Fig. 5. A linear regression fits the data well, without intercept. We conclude that the energy velocity $v_{E}$ $=3 \mathrm{D} / \mathrm{l}$ is wavelength independent in this region.

The observed wavelength independence of $v_{E}$ in a regime where $D$ is strongly wavelength dependent is surprising when compared to existing experimental studies on wavelength-dependent scattering in random media consisting of $\mathrm{TiO}_{2}$ spheres. If the wavelength is tuned in resonance with the $\mathrm{TiO}_{2}$ spheres, the energy velocity is strongly reduced by the long dwell time of the light inside the $\mathrm{TiO}_{2}$ spheres [17]. In macroporous $\mathrm{GaP}$, resonances play apparently no important role as the energy velocity is independent of the wavelength. The strong reduction of the diffusion constant at lower wavelengths is almost solely caused by the decrease of the transport mean free path. The microscopic structure of

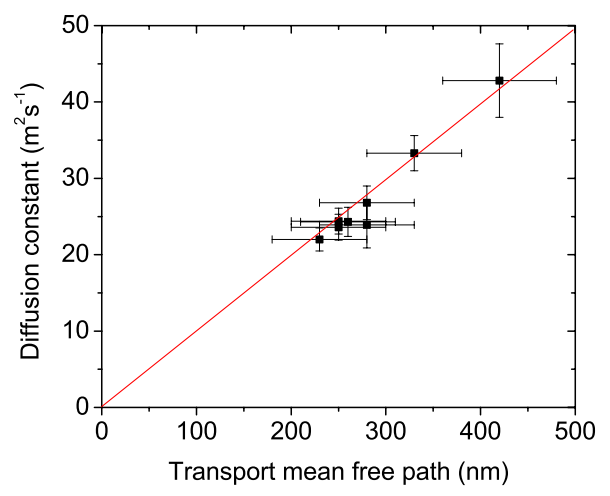

FIG. 5. (Color online) Replot of our measurements of the diffusion constant as presented in Fig. 4 versus transport mean free path. The transport mean free path is varied by changing the wavelength of the light. The uncertainties in the transport mean free path (horizontal) are $\sigma$ errors and are mutually fully correlated $[22,29]$. The straight line indicates a linear relationship between diffusion constant and transport mean free path resulting in a constant energy velocity.

the macroporous $\mathrm{GaP}$ networks contains many randomly shaped subwavelength features [18]. To estimate the wavelength dependence of $v_{E}$ we performed extensive Miescattering calculations and found that none of these small features will show scattering resonances. The absence of these resonances in these inverted structures accounts for the absence of a significant wavelength dependence of $v_{E}$.

From Fig. 5 we find $v_{E} / c=1.0 \pm 0.2$ (uncertainty of one standard deviation) for all wavelengths between $\lambda_{0}$ $=705 \mathrm{~nm}$ and $\lambda_{0}=855 \mathrm{~nm}$. Our measured value for $v_{E}$ is, within the experimental uncertainty, in quantitative agreement with an earlier measurement of $v_{E}$ at $\lambda_{0}=739 \mathrm{~nm}$ performed by Johnson et al. [22]. It is generally believed that $v_{E}$ may never exceed $c$ and it has been shown from microscopic calculations that $v_{E}$ may not exceed the phase velocity in media composed of randomly distributed scatterers $[8,35]$. The phase velocity in porous GaP is $c / n_{\text {eff }}=0.7 \pm 0.1$ where $n_{\text {eff }}$ is the effective refractive index of the medium which is directly related to the extrapolation ratio [36]. We conclude that the value of the energy velocity must be on the lower bound of the error bar. From the linear relation between the diffusion constant and the transport mean free path as well as the high energy velocity we conclude that microscopic resonances are absent in macroporous $\mathrm{GaP}$ between $705 \mathrm{~nm}$ and $855 \mathrm{~nm}$.

To summarize, we have performed time-resolved transmission measurements, with a pulse interferometer technique, on macroporous gallium phosphide at various wavelengths between $705 \mathrm{~nm}$ and $855 \mathrm{~nm}$. We have extracted the diffusion constant from the time-dependent transmitted intensity averaged over different realizations of the random medium. We find that the diffusion constant is strongly wavelength dependent, whereas the energy velocity, related by $v_{E} \equiv 3 D / l$, is not. We conclude that microscopic resonances, which can strongly slow down the diffusion process in, e.g., granular $\mathrm{TiO}_{2}$, are absent in macroporous $\mathrm{GaP}$ within the wavelength region of concern. 
Our measurements can be easily extended to long-time tails by creating free standing porous samples. The timedependent transmission of free-standing porous samples will no longer be affected by after pulses which occur in samples with a substrate. Signs of Anderson localization of light could manifest themselves most prominently in long-time tails.
The authors thank W. L. Vos, R. W. Tjerkstra, P. M. Johnson, and J. Gómez Rivas for valuable discussions, and L. A. Woldering for assistance with sample characterization. This work is part of the research program "Waves in Complex Media" of the Stichting voor Fundamenteel Onderzoek der Materie (FOM), which is financially supported by the Netherlands Organization for Scientific Research (NWO).
[1] T. Schwartz, G. Bartal, S. Fishman, and M. Segev, Nature (London) 446, 52 (2007).

[2] M. Störzer, P. Gross, C. M. Aegerter, and G. Maret, Phys. Rev. Lett. 96, 063904 (2006).

[3] Y. Kuga and A. Ishimaru, J. Opt. Soc. Am. A 8, 831 (1984).

[4] M. P. Van Albada and A. Lagendijk, Phys. Rev. Lett. 55, 2692 (1985).

[5] P. W. Anderson, Phys. Rev. 109, 1492 (1958).

[6] A. F. Ioffe and A. R. Regel, Prog. Semicond. 4, 237 (1960).

[7] S. John, Phys. Rev. Lett. 53, 2169 (1984).

[8] M. P. van Albada, B. A. van Tiggelen, A. Lagendijk, and A. Tip, Phys. Rev. Lett. 66, 3132 (1991).

[9] A. Z. Genack and N. Garcia, Phys. Rev. Lett. 66, 2064 (1991).

[10] A. Z. Genack, P. Sebbah, M. Stoytchev, and B. A. van Tiggelen, Phys. Rev. Lett. 82, 715 (1999).

[11] A. A. Chabanov and A. Z. Genack, Phys. Rev. Lett. 87, 233903 (2001).

[12] D. S. Wiersma, P. Bartolini, A. Lagendijk, and R. Righini, Nature (London) 390, 671 (1997).

[13] F. J. P. Schuurmans, M. Megens, D. Vanmaekelbergh, and A. Lagendijk, Phys. Rev. Lett. 83, 2183 (1999).

[14] C. M. Aegerter, M. Störzer, and G. Maret, Europhys. Lett. 75, 562 (2006).

[15] D. S. Wiersma, M. P. van Albada, B. A. van Tiggelen, and A. Lagendijk, Phys. Rev. Lett. 74, 4193 (1995).

[16] R. H. J. Kop, P. de Vries, R. Sprik, and A. Lagendijk, Phys. Rev. Lett. 79, 4369 (1997).

[17] M. Störzer, C. M. Aegerter, and G. Maret, Phys. Rev. E 73, 065602(R) (2006).

[18] F. J. P. Schuurmans, D. Vanmaekelbergh, J. van de Lagemaat, and A. Lagendijk, Science 284, 141 (1999).

[19] R. W. Tjerkstra, J. G. Rivas, D. Vermaekelbergh, and J. J.
Kelly, Electrochem. Solid-State Lett. 5, G32 (2002).

[20] J. G. Rivas, A. Lagendijk, R. W. Tjerkstra, D. Vanmaekelbergh, and J. J. Kelly, Appl. Phys. Lett. 80, 4498 (2002).

[21] P. M. Johnson, B. P. J. Bret, J. GómezRivas, J. J. Kelly, and A. Lagendijk, Phys. Rev. Lett. 89, 243901 (2002).

[22] P. M. Johnson, A. Imhof, B. P. J. Bret, J. G. Rivas, and A. Lagendijk, Phys. Rev. E 68, 016604 (2003).

[23] R. H. J. Kop and R. Sprik, Rev. Sci. Instrum. 66, 5459 (1995).

[24] I. M. Vellekoop, P. Lodahl, and A. Lagendijk, Phys. Rev. E 71, 056604 (2005)

[25] P. Sebbah, O. Legrand, and A. Z. Genack, Phys. Rev. E 59, 2406 (1999).

[26] B. A. van Tiggelen, P. Sebbah, M. Stoytchev, and A. Z. Genack, Phys. Rev. E 59, 7166 (1999).

[27] A. Z. Genack and J. M. Drake, Europhys. Lett. 11, 331 (1990).

[28] M. U. Vera and D. J. Durian, Phys. Rev. E 53, 3215 (1996).

[29] P. M. Johnson and J. G. Rivas (private communication).

[30] D. F. Nelson and E. H. Turner, J. Appl. Phys. 39, 3337 (1968).

[31] W. L. Bond, J. Appl. Phys. 36, 1674 (1965).

[32] R. Berkovits and M. Kaveh, J. Phys.: Condens. Matter 2, 307 (1990).

[33] S. E. Skipetrov and B. A. van Tiggelen, Phys. Rev. Lett. 96, 043902 (2006).

[34] The measurements of the total transmission versus wavelength can be found in Fig. 5 in Ref. [22]. The linear relationship between transport mean free path and wavelength is concluded from applying Eq. (5) in Ref. [22] to these measurements.

[35] B. A. van Tiggelen, A. Lagendijk, M. P. van Albada, and A. Tip, Phys. Rev. B 45, 12233 (1992).

[36] J. Gomez Rivas, D. H. Dau, A. Imhof, R. Sprik, B. P. J. Bret, P. M. Johnson, T. W. Hijmans, and A. Lagendijk, Opt. Commun. 220, 17 (2003). 